\title{
Neue Verhaltensregeln für Pharmaunternehmen - mit Auswirkungen auf die Ärzteschaft
}

Ab 2016 werden die international tätigen Pharmaunternehmen ihre Zahlungen an Ärzte und Institutionen der Gesundheitsversorgung und -forschung für Beratungsund Dienstleistungen auf ihren Websites offenlegen. Ausserdem gilt für die Pharmaunternehmen ab Mitte 2014 ein grundsätzliches Verbot, Ärzten und anderen Fachpersonen im Gesundheitsbereich Geschenke zu machen. Diese Neuerungen stehen im neuen Pharma-Kooperations-Kodex sowie im revidierten Pharmakodex.

\section{Dieter Grauer}

Dr. iur., Stv. Direktor, scienceindustries (Wirtschaftsverband Chemie Pharma Biotech)
Korrespondenz: Dr. iur. Dieter Grauer scienceindustries Nordstrasse 15 Postfach

CH-8021 Zürich Tel. 0443681728 scienceindustries.ch

\section{Trend zu mehr Transparenz}

Im Verkehr zwischen Pharmaunternehmen und Ärzteschaft mehr Transparenz zu schaffen, entspricht einem internationalen Trend. Er hat seine Wurzeln in den USA. Dort regelt der «Physician Payments Sunshine Act» diese Beziehungen samt Offenlegung der entsprechenden Geldleistungen gesetzlich [1]. In Europa haben Dänemark, Frankreich, Portugal und die Slowakei ähnliche Gesetze schon eingeführt. Nun verfolgt die EU-Kommission europaweit analoge Ziele, dies mit ihrem «Process on Corporate Responsibility in the Field of Pharmaceuticals: Platform on Ethics and Transparency» [2].

Der europäische Verband der pharmazeutischen Industrie (EFPIA) [3] nahm den Ball der EU-Kommission auf und verabschiedete Mitte 2013 den «EFPIA Code on Disclosure of Transfers of Value from Pharmaceutical Companies to Healthcare Professionals and Healthcare Organisations». Danach müssen die Pharmaunternehmen Zahlungen insbesondere an Ärzte, Apotheker, Spitäler und Forschungsinstitutionen offenlegen. Die Offenlegungspflicht ist jährlich zu erfüllen. Sie gilt ab 2016 und erfasst jeweils die im Vorjahr geleisteten Zahlungen. Zugleich geändert wurde der «EFPIA Code of Practice on the promotion of prescription-only medicines to, and interactions with, healthcare professionals». Wichtigste Neuerung ist hier, dass die Pharmaunternehmen ab Mitte 2014 Fachpersonen, insbesondere Ärzten, grundsätzlich keine Geschenke mehr machen dürfen.

\section{Umsetzung auf Länderebene}

Umgesetzt werden die EFPIA Codes [4] durch die nationalen Industrieverbände. Sie müssen den Inhalt der EFPIA-Codes mit nationalen Kodizes konkretisieren und deren Einhaltung überwachen. Jeder EFPIA-Mitgliedverband berücksichtigt beim Erlass der nationalen Kodizes die Rahmenbedingungen, die sich von der nationalen Rechtsordnung sowie von den jeweiligen kulturellen Gegebenheiten und Gewohnheiten her ergeben.

In der Schweiz ist «scienceindustries» dafür zuständig. Koordiniert mit ihren Partnerverbänden hat scienceindustries den neuen Pharma-KooperationsKodex (PKK) ausgearbeitet und den bestehenden Pharmakodex (PK) revidiert. Der PKK regelt die Beziehungen zwischen Pharmaunternehmen und Fachpersonen in der Gesundheitsversorgung (Ärzten, Apotheken), entsprechenden Organisationen (Spitälern, Forschungsinstitutionen) und Patientenorganisationen sowie die Geldflüsse zwischen ihnen. Der bereits seit 2004 geltende und nun teilrevidierte PK regelt Folgendes: Die Fachwerbung für Arzneimittel, die Veranstaltungen zur Arzneimittel-Fachwerbung und -Information sowie zur Weiter- und Fortbildung von Fachpersonen und schliesslich das Sponsoring klinischer Versuche mit Arzneimitteln und Durchführung nicht-interventioneller Untersuchungen. Die neugeschaffenen Integritätsgrundsätze lauten in beiden Kodizes gleich.

Der neue PKK und der revidierte PK sind seit dem 1. Januar 2014 in Kraft [5]. Sie gelten für die Unternehmen, die sich durch Unterzeichnung der entsprechenden Erklärung darauf verpflichtet haben [6]. Für die Aufsicht über den PK und den PKK in der Schweiz ist das bei scienceindustries angesiedelte Kodex-Sekretariat zuständig.

\section{Offenlegung geldwerter Leistungen}

Gemäss dem Pharma-Kooperations-Kodex müssen die Pharmaunternehmen die Zusammenarbeit mit Fachpersonen und Gesundheitsversorgungs-Organisationen vertraglich regeln. Abgeltungen (geldwerte Leistungen) aufgrund solcher Verträge müssen die Pharmaunternehmen ab 2016 auf ihren Unternehmens-Websites jährlich offenlegen. Damit wird transparent, welches Unternehmen welchen Fachpersonen oder Organisationen wofür wie viel be- 


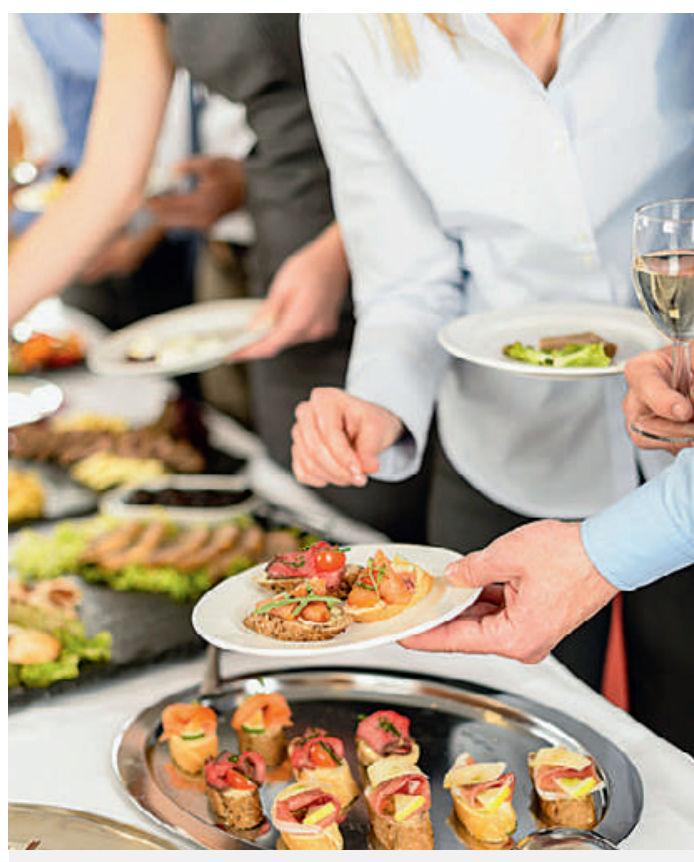

Fällt nicht unter das Geschenkverbot: die Bezahlung von Mahlzeiten und Getränken. Sie ist erlaubt bis maximal 150 Franken pro Person.

zahlt hat, z. B. für Referate, Beratungsgremien, Forschung und Entwicklung. Transparent werden so auch Beiträge an Reise- und Unterkunftskosten im Zusammenhang mit Kongressen oder anderen Veranstaltungen. Von der Offenlegungspflicht ausgenommen sind die Abgeltungen für Fachpersonen bei Bestellungen und Lieferungen von Arzneimitteln,

\section{Transparent werden so auch Beiträge an Reise- und Unterkunftskosten im Zusammenhang mit Kongressen oder anderen Veranstaltungen.}

\section{Interaktiver
Artikel}

Wollen Sie diesen Artike kommentieren? Nutzen Sie dafür die Kommentarfunktion in der OnlineVersion oder sehen Sie nach, was Ihre Kolleginnen und Kollegen bereits geschrieben haben: www.saez.ch/ aktuelle-ausgabe/ interaktive-beitraege/ ebenso die unentgeltliche Abgabe von Mustern verschreibungspflichtiger Arzneimittel sowie die Bezahlung von Mahlzeiten (einschliesslich Getränke). Bei der Form der Offenlegung richten sich die Pharmaunternehmen nach den EFPIA-Vorgaben [7].

Die Pharmaunternehmen weisen die Fachpersonen oder Gesundheitsversorgungs-Organisationen beim Vertragsabschluss auf ihre Pflicht hin, die vereinbarten Abgeltungen offenzulegen. Die Empfänger der Abgeltung müssen der Offenlegung zustimmen. Lehnt eine Fachperson oder Gesundheitsversorgungs-Organisation die Offenlegung ab, so sieht das Pharmaunternehmen vom Vertragsabschluss ab, weil es sich sonst kodexwidrig verhält.

Die Veranstaltungen zur Fort- und Weiterbildung sind im Pharmakodex geregelt. Die Veranstal-
tungs-Teilnehmer müssen grundsätzlich einen Teil der Kosten selber tragen. Begleitpersonen von Veranstaltungs-Teilnehmern dürfen die Pharmaunternehmen keine Reise-, Verpflegungs- und Unterkunftskosten bezahlen. Diese und weitere Pharmakodex-Regeln korrelieren in den Grundzügen mit den SAMW-Richtlinien für die Zusammenarbeit zwischen Ärzteschaft und Industrie [8].

\section{Europaweit geltendes Geschenkverbot}

Parallel zur Offenlegungspflicht auferlegt die EFPIA den Pharmaunternehmen neu ein europaweit gültiges Verbot, den Fachpersonen Geschenke zu machen. Dieses Verbot gilt ab 1. Juli 2014. Die Ausnahmen dazu sind eng umrissen: Erlaubt sind ausschliesslich für Fachpersonen bestimmte Gegenstände, Informations- und Ausbildungsmaterialien von bescheidenem Wert. Diese Objekte müssen der medizinischen oder pharmazeutischen Tätigkeit oder der medizinischen oder pharmazeutischen Fort- und Weiterbildung dienen; zudem müssen sie für die Patienten von Nutzen sein. An Veranstaltungen können die Pharmaunternehmen ausserdem den Teilnehmern Hilfsmittel von bescheidenem Wert (d.h. Schreibgeräte und -blöcke) zur Verfügung stellen. Diese Hilfsmittel dürfen den Namen des Pharmaunternehmens tragen, nicht aber für bestimmte Arzneimittel werben. Nicht unter das Geschenkverbot fällt schliesslich die Bezahlung von Mahlzeiten und Getränken, erlaubt bis maximal 150 Franken pro Person. Allerdings ist dieser Höchstwert an den Grundsatz gebunden, dass auch hier jeweils angemessene Bescheidenheit geboten ist.

\section{Referenzen}

1 http://thomas.loc.gov/cgi-bin/query/z?c111:S.301: http://www.gpo.gov/fdsys/pkg/FR-2013-02-08/ pdf/2013-02572.pdf

2 http://ec.europa.eu/enterprise/sectors/healthcare/ competitiveness/process_on_corporate_responsibility/ platform-ethics/index_en.htm; http://www.eu-patient.eu/Documents/News/List-Guiding-Principles_ Nov2012.pdf

3 www.efpia.eu

4 Alle EFPIA Codes sind hier veröffentlicht: http:// transparency.efpia.eu/the-efpia-code-2

5 Pharma-Kooperations-Kodex und Pharmakodex sind hier veröffentlicht: http://www.scienceindustries.ch/ engagements/pharmakodex

6 Unterzeichnerlisten: http://www.scienceindustries.ch/ engagements/pharmakodex/unterzeichner-des-pharmakodexes; www.scienceindustries.ch/engagements/ pharmakodex/unterzeichner-des-pharma-kooperations-kodexes

7 http://transparency.efpia.eu/EFPIA\%20DISCLOSURE\%20CODE\%20SCHEDULE\%202\%20 Template\%20FINAL.pdf

8 www.samw.ch/de/Ethik/Aerzteschaft-Industrie.html 